\title{
Alimentation, fertilité et bien-être des oiseaux reproducteurs domestiques : des liens complexes
}

\author{
S. BRIERE I, J.-P. BRILLARD ${ }^{2}$, M. PANHELEUX ${ }^{3}$, P. FROMENT ${ }^{4,5,6,7}$ \\ ${ }^{1}$ GRELIER, 11 rue Saint Eloi, F-49290 Saint-Laurent de la Plaine, France \\ ${ }^{2}$ FERTIL'AVI, 10 rue du 8 Mai, F-37360 Rouziers de Touraine, France \\ ${ }^{3}$ CCPA, Service Avicole Cunicole, Parc d'activité du bois de Teillay, F-35150 Janze, France \\ ${ }^{4}$ INRA, UMR85 Physiologie de la Reproduction et des Comportements, F-37380 Nouzilly, France \\ ${ }^{5}$ CNRS, Physiologie de la Reproduction et des Comportements, F-37380 Nouzilly, France \\ ${ }^{6}$ Université François Rabelais de Tours, F-37041 Tours, France \\ ${ }^{7}$ Haras Nationaux, F-37380 Nouzilly, France \\ Courriel : pfroment@tours.inra.fr
}

L'alimentation en aviculture a une grande importance dans la régulation de la croissance et de la reproduction des volailles. Elle valorise la sélection génétique intensive dans ces espèces. Cette revue décrit, chez la volaille reproductrice, les relations complexes entre la nutrition et la fertilité dans les deux sexes tant au niveau de l'organisme que sur les mécanismes cellulaires et moléculaires pouvant être impliqués.

Pour des raisons économiques, la sélection des volailles destinées au marché de la viande a été principalement basée sur le critère de vitesse de croissance exacerbée des descendants. Ainsi, la compétition entre sélectionneurs laisse peu de place à une amélioration d'autres caractères comme celui de la fertilité des poules et des coqs. Malheureusement, la sélection sur la croissance a engendré des reproducteurs très lourds qui sont peu aptes à se reproduire et qui présentent également des perturbations ovarienne et testiculaire. Une méthode utilisée depuis plusieurs décennies pour limiter le poids des animaux reproducteurs et maintenir des performances de reproduction est le rationnement alimentaire. Le rationnement a montré, en partie, son efficacité à maintenir la fertilité des troupeaux tout en minimisant la mortalité liée à un excès d'engraissement (Hocking 2010).

Chez les oiseaux, comme pour d'autres espèces, la nutrition et plus particulièrement le métabolisme énergétique influencent la reproduction. Cependant, les mécanismes qui sous-tendent les relations entre ces deux grandes fonctions restent à ce jour mal connus. Ainsi, les changements de statut nutritionnel et métabolique peuvent être transmis au système reproducteur (hypothalamus, hypophyse et gonades) via les modifications de certaines secrétions hormonales (ex : insuline, adipocytokines...), autant que via la digestion des substrats énergétiques alimentaires : glucose, acides gras et acides aminés. Nous apportons ici plusieurs éléments sur les relations complexes qui s'établissent entre l'ingéré alimentaire, les nutriments et la fonction de reproduction chez l'oiseau. L'impact du rationnement alimentaire sur ces différents paramètres est également abordé.

\section{1 / Effet de l'alimentation sur la capacité de reproduc- tion}

\section{1 / Relation croissance, "sur-} poids» et reproduction chez l'oiseau

Dans les espèces d'intérêt agronomique sélectionnées pour la production de viande, une croissance rapide des individus sélectionnés est presque toujours accompagnée d'une altération des capacités de reproduction maximale dans chacun des deux sexes (pour Gallus domesticus, voir Dunnington et Siegel 1984). Le cas des lignées de poulets de chair est typique : la sélection de lignées à croissance rapide, mise en œuvre depuis plus de 60 ans a abouti à des poulets atteignant initialement $2 \mathrm{~kg}$ en $100 \mathrm{j}(1950)$, puis en $49 \mathrm{j}$ (1985) et enfin en $35 \mathrm{j}$ (2007) !
Une telle augmentation des performances de croissance s'est accompagnée, chez les mâles de ces lignées, d'une très grande précocité sexuelle. Cette grande précocité "spontanée» se traduit par l'apparition de spermatozoïdes testiculaires dès l'âge de 11-12 semaines chez le coq alors que l'entrée en ponte des poules est désynchronisée car elle n'apparaît qu'à 24-25 semaines. Elle se traduit aussi par un développement testiculaire maximum relativement faible et par une régression testiculaire dès l'âge de 43-45 semaines alors que la saison de ponte se termine seulement à 64-65 semaines, etc. De plus, une proportion de plus en plus élevée de ces coqs (de l'ordre de 40 à $60 \%$ des effectifs) présente une saison de reproduction de moins en moins longue, ce qui conduit à les remplacer bien avant la fin de la période de ponte de femelles entraînant parfois des problèmes sévères de comportement social. En 1990, Reddy et Sadjadi estimaient que les mâles avaient une diminution de la capacité à féconder les œufs d'environ $0,5 \%$ à chaque nouvelle génération (figure 1, Hammerstedt 1999). Cependant, une croissance excessive des mâles s'accompagne généralement de comportements hyperphagiques dus à une surconsommation alimentaire par rapport à leurs besoins.

Concernant les femelles, l'augmentation du poids génère un développement 
Figure 1. Etude du nombre de mâles nécessaires pour maintenir la production (1000 femelles) sur la base de données obtenues des producteurs de souches chair de Pennsylvanie (1997) (d'après R. Hammerstedt 1999).

Données associées à une courbe tendance de l'âge des poulets auquel ils atteignent le poids de $2 \mathrm{~kg}$ (jours).

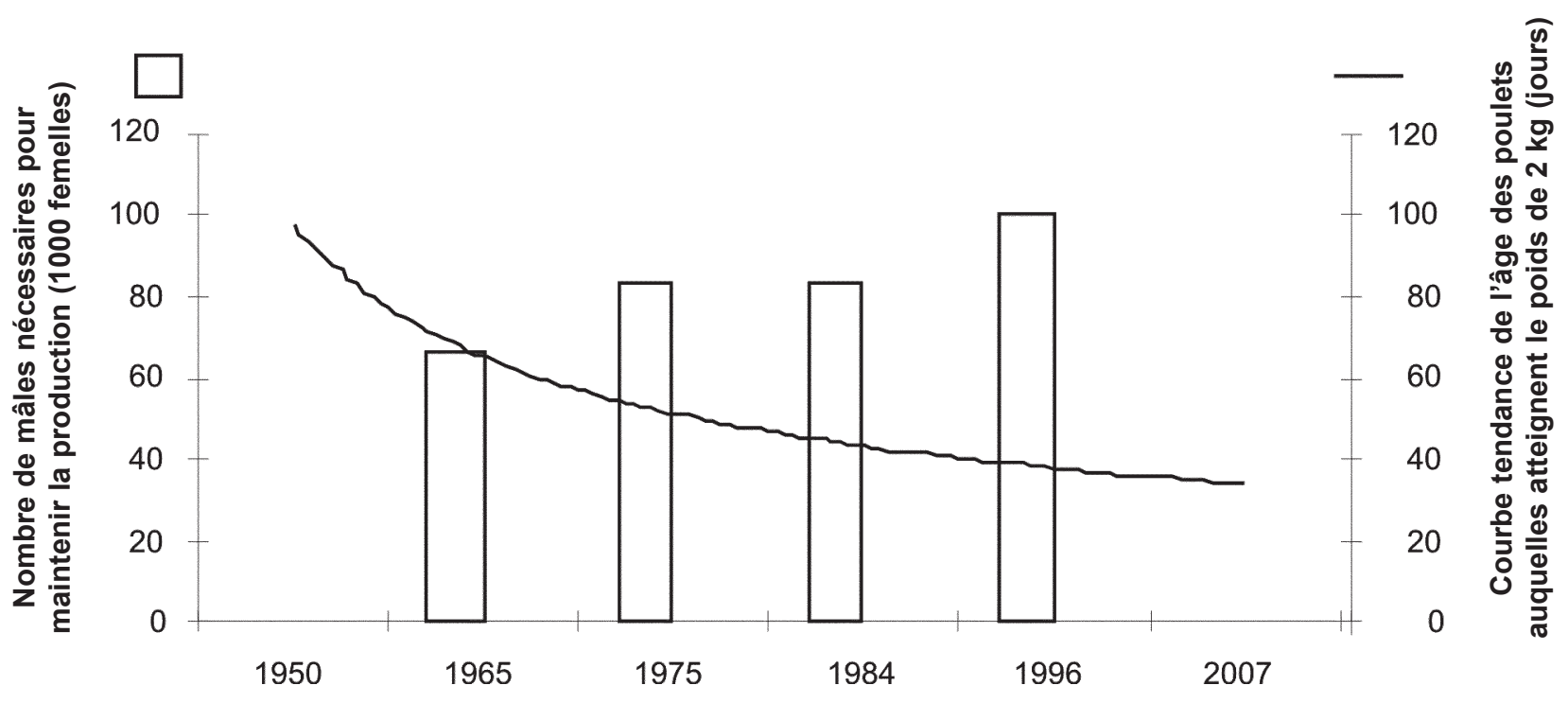

anarchique des follicules pouvant conduire à la coexistence de plusieurs hiérarchies folliculaires qui perturbe les ovulations (Hocking et al 1989). Ce même auteur a par ailleurs montré une relation existant entre le poids des poules à la maturité sexuelle et le nombre de gros follicules en croissance sur l'ovaire (Hocking 1993).

Pour les deux sexes, le maintien de performances de reproduction (ex : ponte et fertilité) conformes au standard de la souche ne peut être assuré qu'à condition d'appliquer dès le très jeune âge (2-3 semaines après l'éclosion) un rationnement alimentaire strict. Ainsi, le contrôle du poids corporel via le rationnement alimentaire permet de conserver sur les mâles : a) une morphologie et une diminution des troubles locomoteurs induits par le surpoids compatibles avec l'accouplement (Leterrier et al 1998); b) une fertilité acceptable au moins pendant la première partie de la saison sexuelle (Hocking 1991, Brillard 2003 et 2004). Il s'avère cependant que l'application d'un rationnement aura des effets secondaires sur le comportement, comme celui du picage (Savory et Maros 1993).

\section{2 / Restriction alimentaire}

Il a été démontré qu'une restriction alimentaire quantitative des mâles pendant leur croissance a pour conséquence de retarder l'âge d'apparition de la puberté (figure 2A), de diminuer la part du coût alimentaire dans les coûts d'élevage et, au total d'augmenter la fertilité des troupeaux (de Reviers 1973 et 1974). Un rationnement quantitatif adapté à chaque lignée maintient de manière assez durable un poids testiculaire constant au cours de la période de reproduction alors que le poids testiculaire régresse dès la 40-45 ème semaine d'âge chez des coqs nourris ad libitum, entraînant indirectement une altération rapide de la fertilité des œufs (de Reviers 1990).

Chez la poule reproductrice chair, une restriction alimentaire de l'ordre de 20$30 \%$ de l'ad libitum retarde l'âge du premier œuf (Dunnington et Siegel 1984, Hester et Stevens 1990). Cependant, une restriction alimentaire quantitative modérée en phase d'élevage a des conséquences bénéfiques sur la fertilité de ces lignées (Yu et al 1992, Renema et al 1999a et b, Hocking et Robertson 2000). Ainsi, une telle restriction (de $-15 \%$ de l'ad libitum par exemple) appliquée chez des poules reproductrices naines se traduit par l'apparition d'une véritable hiérarchie folliculaire stricte (un seul follicule par stade de développement) et optimise les performances de ponte (Hocking 1987) à l'encontre de ce qui est observé chez des poules reproductrices nourries ad libitum (Renema et al 1999b). Chez ces dernières, on observe sur l'ovaire un plus grand nombre de follicules préovulatoires que chez des femelles rationnées. Plusieurs hiérarchies folliculaires sont alors mises en place, augmentant l'activité ovarienne et par conséquence des dysfonctionnements ovulatoires tels que le nombre de doubles ovulations. De telles altérations de la fonction ovarienne sont associées à une baisse du nombre d'œufs pondus chez la poule reproductrice chair mais aussi à une baisse de la qualité de coquille des œufs pondus.

Le contrôle de la mise en place d'une hiérarchie folliculaire analogue à celle observée chez les poules de lignée ponte s'explique en partie par le fait que le rationnement alimentaire s'accompagne d'une réduction de la production de lipides au niveau hépatique, grâce à la diminution de l'expression des gènes impliqués dans la lipogenèse (Richards et al 2003). Une restriction alimentaire marquée contribue à diminuer l'atrésie folliculaire et la production de follicules en phase de croissance rapide ce qui limite l'apparition de doubles ovulations. Au total, la mise en place, chez la poule, d'un rationnement quantitatif en cours d'élevage permet de contrôler le poids des reproductrices et l'apparition de vagues folliculaires multiples tout en retardant l'âge d'entrée en ponte (Hocking et al 1989). Chez la dinde, Crouch et al (2002a, b et c) rapporte une augmentation des performances de ponte $(+8$ œufs par femelle) en mettant en place une réduction de $55 \%$ de la quantité d'aliment distribuée aux futures reproductrices à l'âge de 16 semaines. Ces mêmes auteurs ont également étudié l'incidence de l'âge, de la saison et de la durée de la période de restriction sur les performances de reproduction des dindes. Une restriction initiée trop 
Figure 2. Conséquences d'une alimentation à volonté ou restreinte sur:

A. le développement testiculaire de poulet de souche chair âgé de 8 semaines.

B. le développement de l'ovaire chez les poules reproductrices chair.

C. le taux de ponte (d'après Heck et al 2004).

A

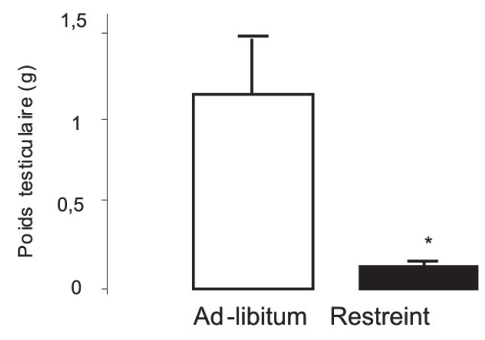

B

Alimentation à volonté
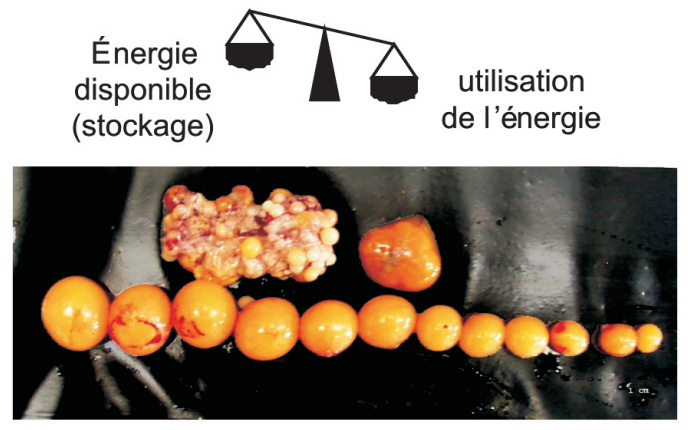

Perte de la hiérarchie folliculaire induisant des ovulations multiples

\section{Restriction alimentaire}

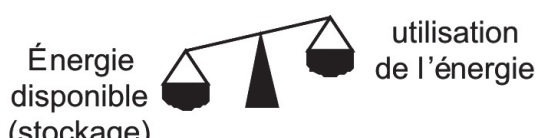

(stockage)

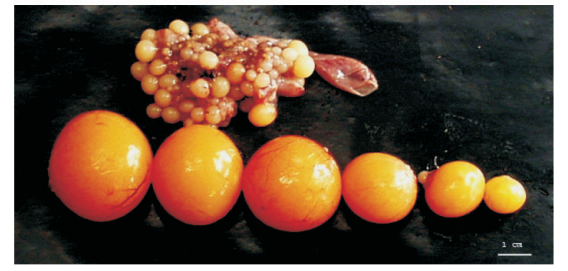

Hiérarchie folliculaire
C

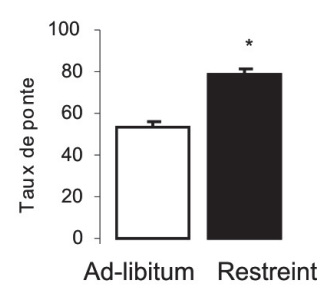

* : moyennes diffèrent significativement $(p<0,05)$.

précocement au cours de la phase d'élevage a un impact beaucoup plus marqué sur l'axe somatotrope et donc sur la croissance des femelles qu'une restriction tardive, favorisant plus tard une hiérarchie folliculaire anarchique préjudiciable (Hocking 2010).

Cependant, les bénéfices évidents apportés par la mise en place de programmes de restriction alimentaire modérée (de l'ordre de 10-15\% de l'ad libitum) chez les poules reproductrices chair et pouvant atteindre $65 \%$ de l' ad libitum pour un jeune mâle en croissance, sont souvent accompagnés de modifications du comportement alimentaire et social tels qu'une augmentation de la consommation d'eau, une hyperactivité, des comportements stéréotypés, d'agressivité et/ou encore l'apparition de picage (Kostal et al 1992, MacLeod et al 1993, Savory et Mann 1999).

\section{3 / Suralimentation}

Chez les oiseaux, la fonction du foie présente quelques spécificités par rapport aux mammifères. Lors d'une prise alimentaire, les lipides absorbés au niveau de l'intestin vont traverser d'abord le foie où ils peuvent être captés et être utilisés avant de rejoindre la circulation sanguine (Hermier et al 1999). De plus, l'apport de glucides alimentaires va stimuler la synthèse lipidique. Le foie, chez les oiseaux, est d'ailleurs le site principal de la lipogenèse de novo notamment de la synthèse des triglycérides, mais aussi, comme chez les mammifères, des phospholipides et du cholestérol (Leveille et al 1975). Ces triglycérides produits par le foie sont soit incorporés dans les VLDL (Very Low Density Lipoprotein) puis transportés par le sang notamment vers les ovocytes en croissance, les adipocytes et le tissu musculaire, soit peuvent être stockés dans le foie. Un cas bien connu, le foie gras chez les palmipèdes (oie, canard), est lié à un stockage excessif de lipides dans le foie provoquant une stéatose hépatique «naturelle» qui, à l'inverse des mammifères, peut retrouver son aspect «normal» après une période de jeûne (Hermier et al 1999). Cette «réversibilité» est une spécificité des oiseaux migrateurs (ex: les palmipèdes), puisque cette stéatose hépatique physiologique fournit de l'énergie pendant les jours de migration (Bénard et Labie 1998). Chez les oiseaux, le foie produit également la vitellogenine qui agit sur la fonction ovarienne. L'ovaire peut aussi interagir sur l'activité hépa- tique, puisque aussi bien la production de vitellogenine que des VLDL hépatiques sont stimulés par les œstrogenes (Davis 1997).

Bien que chez les volailles il n'y a pas de véritable obésité contrairement aux mammifères, le surpoids observé est plutôt associé aux lignées à croissance rapide (souche viande) qui peuvent avoir un comportement d'hyperphagie. Comme décrit ci-dessus, l'efficacité de la sélection a conduit à avoir une croissance rapide tout en minimisant les dépenses énergétiques (figure 1), ce qui suggère une gestion du métabolisme différente des autres lignées comme les lignées pontes. Toutefois, des modèles d'oiseaux en surpoids (nourriture $a d$ libitum ou gavage) ont permis de mieux comprendre les conséquences que peut avoir une suralimentation sur la fonction de reproduction dans les deux sexes. Ainsi, chez le coq, une obésité induite par gavage diminue la production de spermatozoïdes de $50 \%$ et chez la poule réduit la production d'œuf. Chez le mâle, cette baisse de fertilité est la conséquence d'une diminution du poids testiculaire (environ - $30 \%$ en 4 semaines), qui s'accompagne d'une baisse de la testostéronémie et d'une augmentation des concentrations intra- 
testiculaires en cholestérol ainsi que d'une augmentation de la température interne de $0,3^{\circ} \mathrm{C}$ (Nir et al 1975). L'une des hypothèses avancées pour expliquer ces modifications de la thermorégulation serait que les oiseaux ne possèdent pas, contrairement aux mammifères, de complexe pampiniforme (complexe veineux et artériel qui permet de maintenir constante la température intra-testiculaire). Ainsi, l'augmentation de la température consécutive à l'augmentation d'énergie résultant du gavage conduirait à une altération de l'état fonctionnel des spermatogonies souches, entraînant par là-même une diminution de la production de spermatozoïdes.

Chez la femelle immature, un poids corporel important est souvent la conséquence d'une consommation excessive comparativement à leurs besoins, ce qui provoque un développement accéléré de l'appareil reproducteur au moment de la maturité sexuelle (Wilson et Harms 1986, Yu et al 1992) et une hyperactivité ovarienne. Bien que la production d'un œuf soit un processus nécessitant beaucoup d'énergie, un excès d'énergie diminue la production d'ovocytes fonctionnels en provoquant une dérégulation de la hiérarchie folliculaire (Renema et al 1999b) (figure 2). Une augmentation de la fréquence des ovulations multiples ou d'ovulation rapprochées conduit notamment à une plus grande incidence d'œufs anormaux (déformés, mous...). Les ovulations multiples entraînent l'apparition «d'œufs doubles») (= à doubles jaunes) alors que les ovulations rapprochées conduisent généralement à l'apparition d'un premier œuf «normal», le second étant alors plus petit, déformé et présentant des défauts de calcification (Walzem et al 1993). Walzem et al (1994) ont étudié l'effet d'une suralimentation sur la production lipoprotéique hépatique en prenant comme modèle la poule pondeuse. Ils ont observé une augmentation du diamètre des vésicules lipidiques de type VLDL qui ont la particularité chez la poule d'avoir une taille identique d'environ $30 \mathrm{~nm}$. Cette altération de leurs propriétés physicochimiques modifient leur transport sanguin jusqu'au(x) follicule(s) en cours de développement. A terme, ce défaut de transport conduit à un arrêt des dépôts de vitellus dans les follicules en croissance. Chez de telles femelles, un rationnement quantitatif modéré ou bien une limitation de l'apport énergétique alimentaire (restriction «qualitative») est en général suffisant pour retrouver un taux d'ovulation optimal (Hocking 1987, Yu et al 1992, Renema et al 1999a).

\section{4 / De nutriments spécifiques pour satisfaire les besoins de reproduction}

\section{a) Action des lipides}

Chez les mammifères, plusieurs modèles de souris transgéniques ont montré l'importance du métabolisme lipidique dans la fertilité mâle et femelle. En effet, l'inhibition ou l'invalidation de gènes régulant l'homéostasie du cholestérol et des acides gras (récepteur nucléaire Liver $X$ Receptor, Peroxysome Proliferator Activated Receptor gamma, Lipase Hormono-sensible, AMP-Activated Protein Kinase) conduisent à une altération de la spermatogenèse et de la stéroïdogenèse ovarienne (Robertson et al 2005). De plus, chez le coq, une augmentation de l'apport en acide gras polyinsaturés (AGPI) de type n-3 dans la ration alimentaire permet d'augmenter la qualité de la semence (Blesbois et al 1997). Chez le rat, il a été montré que la synthèse des AGPI à longue chaîne, présents dans les membranes cellulaires des spermatozoïdes, est assurée par la transformation des AGPI essentiels n-3 et n- 6 par les cellules nourricières des cellules germinales (cellules de Sertoli) (Retterstol et al 2001). Chez la poule, les lipides présents notamment au niveau de l'ovocyte proviennent soit de l'alimentation (absorption intestinale), soit de la synthèse lipidique au niveau du foie, qui contrairement aux mammifères, est importante, via le métabolisme du glucose (voie de l'Acetyl CoA). Les lipides seront transférés au sein de l'ovocyte grâce aux lipoprotéines de très basse densité (VLDL $\gamma$ ) dont les caractéristiques sont spécifiques de la poule en production. Ainsi, un régime riche en acide gras va augmenter le volume de l'œuf (Houston 1997). L'acide arachidonique (qui est présent dans les VLDL $\gamma$ ) stimule la sécrétion de progestérone par les cellules de la granulosa $\mathrm{de}$ poule (cellules du follicule) (Hertelendy et al 1992). Chez la vache, un résultat similaire a été décrit puisque les cellules de la granulosa obtenues à partir de vache ayant reçu une alimentation supplémentée en acide gras montrent une augmentation du cholestérol intra-folliculaire et de la sécrétion de progestérone in vitro (Wehrman et al 1991). Ces résultats suggèrent que la ration alimentaire riche en acides gras affecte la fonction gonadique aussi bien chez le mâle que chez la femelle.

\section{b) Action des acides aminés et des} protéines

Chez la poule, une étude conduite par Menge (1967) met en évidence l'importance de la source de protéines utilisée dans la fabrication des aliments. En comparant des aliments préparés à partir de céréales semi-purifiées ou purifiées, de meilleurs résultats (ponte, éclosion et poids des œufs) sont obtenus pour les aliments semi-purifiés, mettant en évidence le fait que la composition en protéines exerce une amélioration des performances de reproduction. Une augmentation de 3\% du taux de protéines chez des poules reproductrices (18 vs $15 \%$ ) améliore la ponte de $4 \%$ (Bradley et al 1972). Toutefois, un excès de protéines au cours de la phase de production provoque une baisse des résultats de ponte et d'éclosabilité chez la poule ainsi qu'une réduction de la fertilité des coqs (Pearson et Herron 1981, Sauveur 1988). A l'inverse, au cours de l'élevage, une baisse drastique du taux de protéines à $10,2 \%$ provoque un retard de la maturité sexuelle (augmentation de l'âge d'entrée en ponte) sans toutefois avoir d'impact négatif sur les performances finales (Voitle et al 1973).

Malgré l'obtention de bons résultats chez la poule lors de la mise en place d'un rationnement protéique, il s'avère que les effets mesurés sont inférieurs à ceux obtenus via le rationnement énergétique, qui doit être privilégié. De plus, ce type de restriction nutritionnelle pose à nouveau des questionnements de bienêtre animal puisque une baisse drastique du taux protéique peut conduire, tout comme le rationnement quantitatif, à l'apparition de comportement de picage et de cannibalisme (Cain et al 1984, Ambrosen et Petersen 1997).

Enfin, il est intéressant de noter que depuis les directives liées à l'épizootie d'encéphalopathie spongiforme bovine, l'apport en protéines est d'origine végétale et principalement de soja et non d'origine animale. Cet apport de soja apporte également des polyphénols qui possèdent pour certains comme la génistéine des effets œstrogéniques. Contrairement aux mammifères, les oiseaux sont plus sensibles aux œstrogènes qui peuvent agir, à des doses importantes, sur le comportement sexuel et la reproduction adulte. L'exemple du diamant mandarin qui se nourrit de graines, présente des modifications de son chant, et de la reproduction si les graines ont une concentration en œstrogènes importantes (liée notamment à un épisode de sécheresse et qui concentre ces molécules astrogéniques). De plus, au niveau du foie, les œstrogènes stimulent l'expression des enzymes impliquées dans la synthèse des lipides. Nous pouvons nous poser la question d'une augmentation de l'activité œstrogénique par le biais d'une alimentation riche en soja et les conséquences sur la fonction de reproduction. Toutefois, à l'inverse du diamant 
mandarin, les études chez le poulet suggèrent que les doses en polyphénols ne sont pas assez fortes pour altérer la reproduction.

\section{2 / Mécanismes de régula- tion de la fertilité par l'ali- mentation}

\section{1 / Perception de la balance énergétique}

Dans les cas où la perturbation des apports énergétiques conduit à des troubles de l'ovulation (ex : restriction alimentaire sévère ou alimentation excessive), on observe fréquemment des altérations fonctionnelles du système hypothalamo-hypophysaire et particulièrement de la sécrétion de l'hormone lutéinisante (LH). Cependant, les mécanismes responsables de ce contrôle nutritionnel sur la fonction de reproduction ne sont pas clairement définis chez l'oiseau. Deux types de signaux, hormonaux ou les métabolites issus de la digestion, semblent jouer un rôle intermédiaire entre le bilan énergétique et le fonctionnement de l'axe gonadotrope. a) Les hormones, facteurs de croissance et neuromédiateurs

Plusieurs organes comme le tissu adipeux, le foie, le muscle, le duodénum, produisent des signaux hormonaux qui informent l'organisme de la disponibilité en énergie (ex : adipocytokines produites par le tissu adipeux, le facteur de croissance insulin-like growth factor 1 , IGF1, produit par le foie, la ghreline produite par le gésier...) (figure 3).

Chez la dinde, Sun et al (2006) ont rapporté une implication probable de l'axe somatotrope (et notamment l'hormone de croissance Growth Hormone (GH) et l'IGF1) chez les femelles rationnées en cours d'élevage puisque les secrétions de GH et d'IGF1 sont augmentées après le début du rationnement. Or, ces hormones interviennent, au niveau ovarien, dans la régulation de la synthèse des stéroïdes, l'ovulation mais aussi le recrutement et la croissance folliculaires.

De plus, ces signaux hormonaux possèdent des récepteurs spécifiques le long de l'axe hypothalamo-hypophysaire-gonadique (figure 3 ). Un des sites riches en ces récepteurs est l'hypothalamus. Il est composé de plusieurs noyaux de neurones qui interagissent entre eux et régulent différents mécanismes physiologiques comme le métabolisme énergétique, le comportement alimentaire, l'osmolarité, la thermogenèse et la reproduction au travers les neurones secrétant la Gonadotrophine-Releasing Hormone (GnRH), un facteur hypothalamique majeur responsable du contrôle de la sécrétion de gonadotrophines (hormones folliculo stimulante, FSH et LH) qui stimulent directement l'activité des gonades. Depuis la dernière décennie, un autre neuropeptide hypothalamique, le kisspeptine, a été identifié comme un peptide clé dans le contrôle des fonctions de reproduction, et dans le déclenchement de la puberté (Saldanha et al 2010). Il est intéressant de noter que les fonctions de prise alimentaire et de reproduction semblent antagonistes et que les neurones à GnRH et à kisspeptine seraient à la confluence de ces voies de régulation.

Ainsi, chez les mammifères, des inhibiteurs du comportement de prise alimentaire comme le glucose, l'insuline ou la leptine sont plutôt des stimulants

Figure 3. Implications des facteurs endocriniens et nutritionnels dans la régulation de la fonction de reproduction.

\section{Axe gonadotrope}

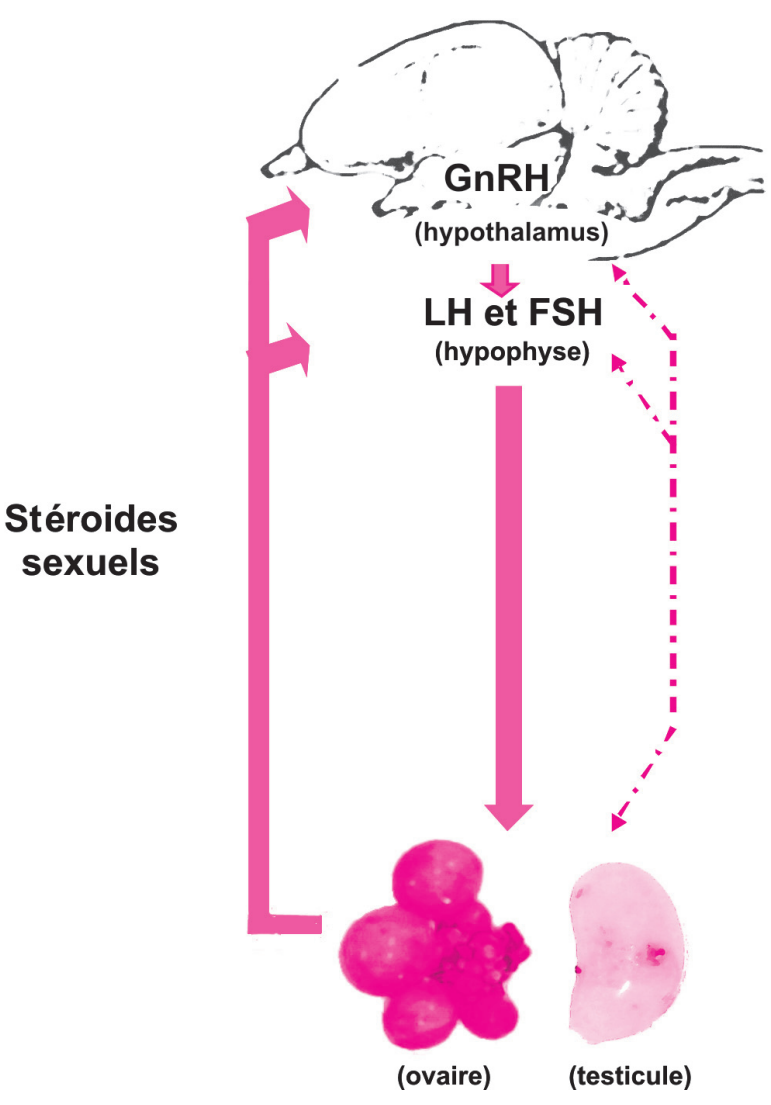

Système digestif

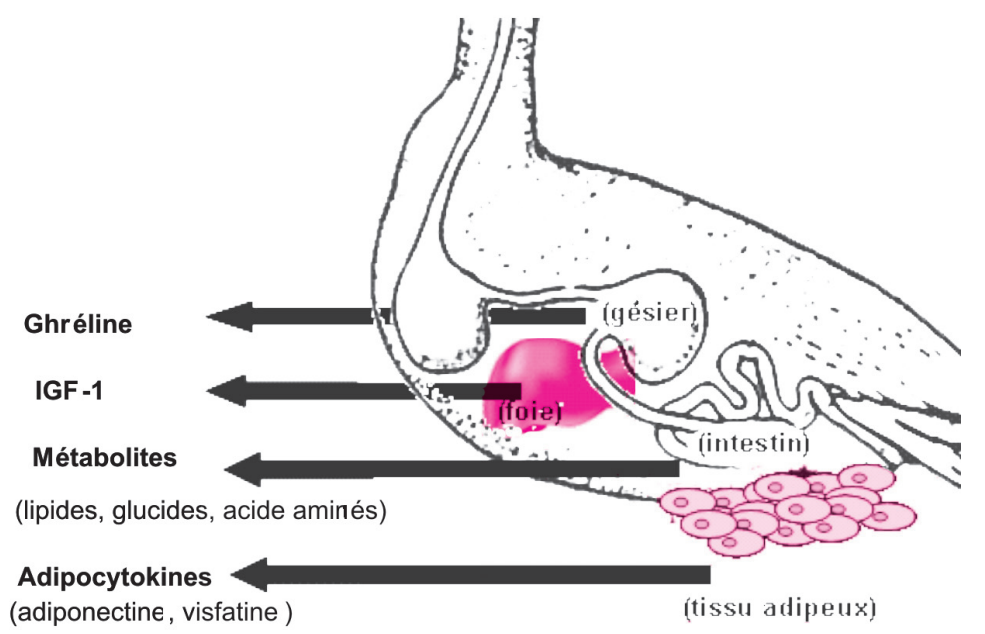


de la fonction de reproduction. Inversement, des stimulateurs de la prise alimentaire comme la ghréline et l'orexine, inhibent la sécrétion des gonadotrophines (Furuta et al 2001). Nous pouvons les séparer en deux catégories, les hormones orexigènes (la ghréline et l'orexine, Furuta et al 2001) qui stimulent la prise alimentaire et inhibent la sécrétion des gonadotrophines, et les hormones anorexigènes (la cholecystokinine, Schreihofer et al 1993) qui ont un effet de satiété et stimulent la sécrétion de LH. La majorité de ces hormones citées sont présentes chez le poulet.

Au centre de ces régulations hormonales au niveau central, le neuropeptide $Y$ (NPY) joue un rôle majeur puisqu'il agit dans la médiation des effets orexigènes (ex. ghréline) et anorexigènes (ex. leptine) chez les rongeurs (Mondal et al 2005). Chez les oiseaux, le NPY est également exprimé au niveau hypothalamique et pourrait avoir des effets similaires.

L'hypothalamus est donc capable, en fonction des ressources énergétiques disponibles, de «diriger» les fonctions prioritaires de l'organisme. Ainsi, lorsque la balance énergétique est négative, la stimulation de l'ingéré alimentaire sera privilégié et les fonctions de reproduction sont mises au repos.

Exemple de signaux hormonaux récemment étudiés : la ghréline et la leptine-like

Identifiée chez le poulet en 2002, la ghréline est une hormone secrétée par le tractus digestif. Son taux augmente avant les repas et diminue après ceuxci. L'expression de la ghréline et son action sur les gonades à travers son récepteur ont été rapportées chez l'humain, le rat et l'oiseau. En effet, chez la poule et la caille, une expression élevée de la ghréline a été observée dans l'ovaire, l'oviducte, le vagin et l'utérus. In vivo, une injection intra-cérébroventriculaire de ghréline chez le poulet inhibe la prise alimentaire (Kaiya et al 2009) (chez les rongeurs ou chez le poisson rouge, la ghréline va stimuler la prise alimentaire : Wren et al 2001, Miura et al 2006). Chez les rongeurs, la ghréline inhibe la sécrétion pulsatile de la GnRH (gonadolibérine), de la LH, et retarde la puberté chez le mâle. Les fonctions régulatrices de l'appétit de cette hormone restent toutefois très peu étudiées chez l'oiseau. En revanche, la ghréline est un puissant régulateur de la fonction ovarienne chez la poule puisqu'elle stimule la prolifération des cellules de la granulosa, diminue la mort cellulaire et module la sécrétion des stéroïdes sexuels (Sirotkin et al 2006).

Chez les mammifères, la leptine est une hormone endocrine produite majoritairement par les cellules du tissu adipeux qui induit la satiété. Elle constitue un indicateur du niveau des réserves adipeuses (Cunningham et al 1999). Chez l'oiseau, bien que l'existence du gène de la leptine soit soumise à controverse, des effets leptine-like ont été rapportés et seraient à l'interface entre nutrition et reproduction. L'expression du récepteur de la leptine existe aussi bien au niveau du système nerveux central qu'au niveau de l'ovaire de poule. Ainsi, chez la femelle, l'injection de leptine stimule la folliculogenèse (Song et al 2009), et l'immunisation des poules contre la leptine de poulet présentent une diminution du taux de ponte de $50 \%$ après 60 jours d'immunisation (Shi et al 2006). L'injection de $0,1 \mu \mathrm{g}$ de leptine murine dans l'œuf de caille conduit à l'âge adulte à avoir chez le mâle des concentrations en testostérone sériques deux fois plus importantes que les individus contrôles (Lamosová et al 2003).

\section{b) Régulation par les métabolites}

Les nutriments (glucose, lipides, acides aminés) envoient directement dans la cellule des signaux au niveau de l'ADN pour informer du niveau des réserves énergétiques restantes dans l'organisme. Ces signaux sont des récepteurs nucléaires (ex : PPAR) ou des facteurs de transcription (ex : SREBP, ChREBP) activés par des acides gras ou du glucose qui agissent directement au niveau du gène. Une autre classe de protéines, est plutôt sensible aux acides aminés ou directement au stock énergétique (ATP), ce sont des protéines kinases (complexe mTOR, AMPK, et Pas-Kinase, figure 4) (Grose et al 2009). Chez le poulet, certaines de ces protéines kinases sont exprimées dans les gonades et au niveau central (données personnelles) et leur activation modifierait la fonction de

Figure 4. Relation entre les protéines kinases mTOR, AMPK et PAS-Kinase dans le contrôle de la balance énergétique à partir des régulations montrées chez les mammifères.

en noir non grisé : hormones qui régulent les protéines kinases, en rouge : métabolites qui régulent les protéines kinases.

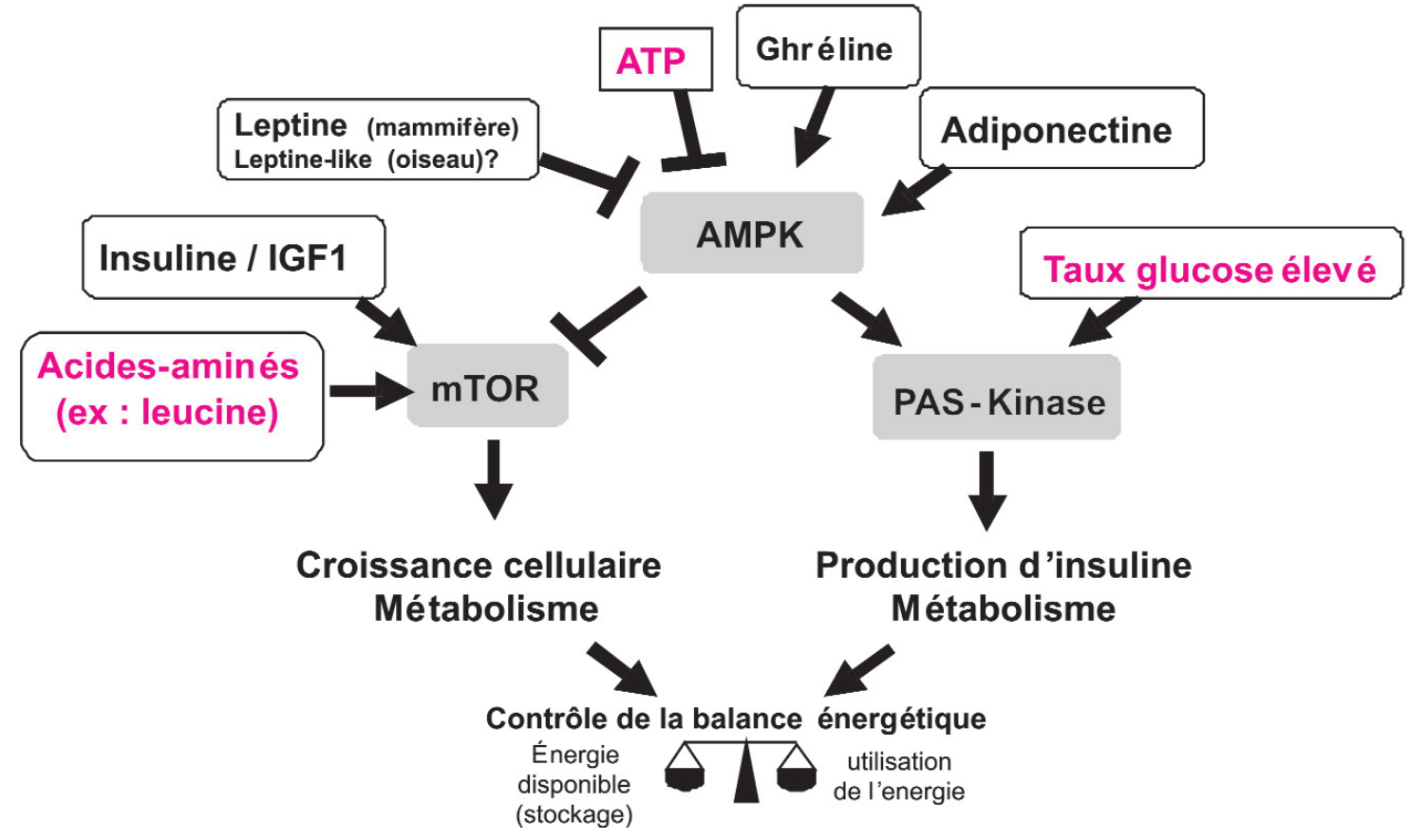


reproduction. Par exemple, in vitro, l'activation de l'AMPK (une protéine sensible au déficit énergétique) diminue l'effet stimulateur de la FSH dans les cellules ovariennes de poule (Tosca et al 2006). In vivo, chez le poulet, l'administration d'un activateur de l'AMPK dans l'eau de boisson, retarde la puberté en agissant aussi bien sur les cellules qui produisent la testostérone que sur les cellules qui nourrissent les cellules germinales (données personnelles).

\section{2 / Quelles voies nutritionnelles envisagées pour améliorer le bien-être des reproducteurs ?}

Des études, réalisées sur des petits groupes, ont démontré que les reproducteurs rationnés (quantitativement) sont plus actifs (exploration litière, consommation eau) et présentent une plus grande attractivité pour l'aliment (Savory et Maros 1993, Puterflam et al 2006). Ces observations soulignent les conséquences du rationnement sur le bien-être animal (frustration, notion de faim...).

En parallèle aux études visant à contrôler le poids des futurs reproducteurs par rationnement quantitatif, une réflexion sur la (les) possibilité(s) d'instaurer un rationnement qualitatif s'est donc développée (de Jong et al 2005). L'apport d'aliment «riches en fibres» est la voie d'étude privilégiée. Cette voie a effectivement déjà fait l'objet d'étude en poule pondeuse afin de réduire les comportements de picage et de cannibalisme. Plus précisément, Hocking et al (2004) ont confirmé que l'ajout de quantités croissantes de fibres apportées par différents matières premières (coque d'avoine, tourteau de tournesol et pulpe de betterave) réduit partiellement la croissance des reproductrices et modifie les comportements de picage identifiés comme expression de stress (picage) avec des conséquences favorables en termes de cannibalis- me. La maîtrise du poids via l'ajout de fibre est donc possible. La variabilité mesurée est par contre importante dans certaines études ce qui est préjudiciable (Savory et al 1996). De plus, les effets mesurés sur les indicateurs de bien-être sont variables selon les études (Savory et al 1996, de Jong et al 2002 et 2005). La méthode de présentation de l'aliment (taille des particules alimentaires) ou de distribution de l'alimentation sur une large zone circulaire (méthode de canon à dispersion de la nourriture, dit «spin feeder»)) joue un rôle puisqu'il peut réduire la compétition et le stress au moment des repas et ainsi améliorer le bien-être. Des suppresseurs d'appétit tel que le propionate de calcium ont également fait l'objet d'études (Tolkamp et al 2005). Leur utilisation est difficile à mettre en œuvre notamment pour des questions d'acceptation par le consommateur (Hocking 2009).

Enfin, la combinaison de matières premières, de présentation de l'aliment, et d'utilisation de la dilution énergétique permet d'ouvrir sur de nouvelles possibilités pour améliorer le bien-être.

\section{3 / Vers la sélection de nouvel- les lignées génétiques ?}

L'évolution génétique des souches de poulet dites «standard» est telle que leur poids a plus que doublé durant les 30 dernières années. Les performances de reproduction des souches sont négativement corrélées à cette évolution (Reddy et Sajadi 1990). Qualité de la semence et ovulations multiples sont les principaux soucis rencontrés avec les lignées actuelles non soumises à une restriction alimentaire. Des essais réalisés avec des croisements label, poulet standard et lignées expérimentales (avec le gène du nanisme dwarf) ont mis en évidence, en phase poulette, des profils hormonaux différents entre les génotypes et selon les pratiques d'alimentation ( $A d$ libitum/rationné) (Heck et al 2004). Les interactions hormonales (IGFs, hormones thyroïdiennes...) sont différentes entre génotypes et pratiques d'alimentation. Ces interactions pourraient expliquer les différences de hiérarchies folliculaires et de performances de reproduction.

\section{Conclusion}

Le bien-être : une notion complexe à intégrer en élevage des reproducteurs.

Il est indéniable que les oiseaux restreints connaissent la «sensation de faim» dans le sens où ils consommeraient plus s'ils avaient la possibilité de le faire. Cependant, il est également reconnu que des poulets restreints sont assurément en meilleure santé que les poulets nourris ad libitum avec des aliments aux densités nutritionnelles classiquement pratiquées (Hocking 2009). Des méthodes alternatives au rationnement quantitatif sont envisageables telles que la dilution de l'aliment. L'utilisation des fibres réduit de 3 à $6 \%$ le poids des animaux à 15 semaines tout en maintenant l'homogénéité du troupeau (Hocking et al 2004). L'encombrement du tractus digestif généré par les fibres devrait agir sur la sécrétion d'hormones telles que la ghréline dont l'action a été décrite ci-dessus. Ce premier pas est intéressant même s'il reste insuffisant pour les lignées «chair» actuelles.

On peut également envisager, à l'avenir, l'utilisation de compléments alimentaires naturellement présents dans certains végétaux qui activeraient au niveau moléculaire des détecteurs de l'état énergétique (tels que mTOR, AMPK). Une hypothèse serait qu'en agissant sur la qualité de l'alimentation, on pourrait limiter le comportement de frustration et contribuer aussi à limiter l'hétérogénéité inter-individuelle sur les caractères de fertilité.

\section{Références}

Ambrosen T., Peterson V., 1997. The influence of protein level in the diet on cannibalism and quality of plumage of layers. Poult. Sci., 76, 559-563.

Bénard G., Labie C., 1998. Evolution histologique du foie des palmipèdes au cours du gavage. 3 èmes Journ. Rech. Palmipèdes à Foie gras, Bordeaux, 27 au 27 octobre, 31-36.

Blesbois E., Lessire M., Grasseau I., Hallouis J.M., Hermier D., 1997. Effect of dietary fat on the fatty acid composition and fertilizing ability of fowl semen. Biol. Reprod., 56, 1216-1220.

Bradley J., Atkinson R., Quisenberry J., 1972. Relationship of protein level and grain type to turkey reproduction. Poult. Sci., 51, 1786.

Brillard J.P., 2003. Practical aspects of fertility in poultry. World's Poult. Sci. J., 59, 441446.

Brillard J.P., 2004. Natural mating in broiler breeders: present and future concerns. World's Poult. Sci. J., 60, 439-445.

Cain J., Weber J., Lockamy T., Creger C., 1984. Grower diets and bird density effects on growth and cannibalism in ring-necked pheasants. Poult. Sci., 63, 450-457.

Crouch A., Grimes J., Christensen V., Krueger K., 2002a. Effect of physical feed restriction during rearing on large white turkey breeder hens: 1. Growth performance. Poult. Sci., 81, 9-15.

Crouch A., Grimes J., Christensen V., Krueger K., 2002b. Effect of physical feed restriction during rearing on large white turkey breeder hens: 2. Reproductive performance. Poult. Sci., 81, 16-22.

Crouch A., Grimes J., Christensen V., Krueger K., 2002c. Effect of physical feed restriction during rearing on Large White turkey breeder hens: 3. Body and carcass composition. Poult. Sci., 81, 17921797.

Cunningham M.J., Clifton D.K., Steiner R.A., 1999. Leptin's action on reproductive 
axis: perspectives and mechanisms. Biol. Reprod., 60, 216-222.

Davis R.A., 1997. Evolution of processes and regulators of lipoprotein synthesis: from birds to mammals. J. Nutr., 795S-800S.

de Jong I.C., Guémené D., 2011. Major welfare issues in broiler breeders. World's Poult. Sci. J., 67, 73-82..

de Jong I.C., Van Voorst S., Ehlhardt D.A., Blokhuis H.J., 2002, Effects of restricted feeding on physiological stress parameters in growing broiler breeders. Brit. Poult. Sci., 43, 157-168.

de Jong I.C., Enting H., van Voorst A., Blokhuis H.J., 2005. Do low density diets improve broiler breeder welfare during rearing and laying? Poult Sci., 84, 194-203.

de Reviers M., 1973. Le développement testiculaire chez le coq. III. Influence de la durée quotidienne d'éclairement sous photopériodes constantes. Ann. Biol. Anim. Bioch. Biophys., 14, 591-600.

de Reviers M., 1974. Le développement testiculaire chez le coq. IV. Etude quantitative des cellules germinales du coq place sous des photopériodes croissantes appliquées à des âges différents. Ann. Biol. Anim. Biochem. Biophys., 15, 633-641.

de Reviers M., 1990. Effets du rationnement alimentaire chez le coq de type chair. INRA Prod. Anim., 3, 21-30.

Dunnington E.A, Siegel P.B., 1984. Age and body weight at sexual maturity in female white Leghorn chickens. Poult. Sci., 63, 828-830.

Furuta M., Funabashi T., Kimura F., 2001. Intracerebroventricular administration of ghrelin rapidly suppresses pulsatile luteinizing hormone secretion in ovariectomized rats. Biochem. Biophys. Res. Commun., 288, 780 785 .

Grose J.H., Sundwall E., Rutter J., 2009. Regulation and function of yeast PAS kinase: a role in the maintenance of cellular integrity. Cell Cycle, 15, 1824-1832.

Hammerstedt R.H., 1999. Symposium summary and challenges for the future. Poult. Sci. 78, 459-466.

Heck A., Onagbesan O., Tona K., Metayer S., Putterflam J., Jego Y., Trevidy J.J., Decuypere E., Williams J., Picard M., Bruggeman V., 2004. Effects of ad libitum feeding on performance of different strains of broiler breeders. Brit. Poult. Sci., 45, 695-703

Hermier D., Salichon M.R., Guy G., Peresson R., Mourot J., Lagarrigue S., 1999. La stéatose hépatique des palmipèdes gavés : bases métaboliques et sensibilité génétique. INRA Prod. Anim., 12, 265-271

Hertelendy F., Todd H., Molnár M., 1992. Influence of chicken and human lipoproteins on steroidogenesis in granulosa cells of the domestic fowl (Gallus domesticus). Gen. Comp. Endocrinol., 85, 335-340.

Hester P.Y., Stevens R.W., 1990. Feed restriction of turkey breeder hens- a review. Poult. Sci., 69, 1439-1446.

Hocking P.M., 1987. Nutritional interactions with reproduction in birds. Proc. Nutr. Soc., 46, 217-225.

Hocking P.M., 1991. Effects of controlling body weight on the semen production of large white turkey males. Brit. Poult. Sci., 32 , 211-218.

Hocking, P.M. 1993. Effects of body weight at sexual maturity and the degree and age of restriction during rearing on the ovarian follicular hierarchy of broiler breeder females. Brit. Poult. Sci., 34, 793-801.

Hocking P.M., 2009. Biology of breeding poultry. Poult. Sci. Symp., Series. Editions Hocking P.M., CAB International, 29, 307330.

Hocking P.M., 2010. Developments in poultry genetic research 1960-2009. Brit. Poult. Sci., 51, Suppl 1, 44-51.

Hocking P., Robertson G., 2000. Ovarian follicular dynamics in selected and control (relaxed selection) male- and female-lines of broiler breeder fed ad libitum or on restricted allocations of food. Brit. Poult. Sci., 41, 229-234.

Hocking P., Waddington D., Walker D., Gilbert A., 1989. Control of the development of the ovarian follicular hierarchy in broiler breeder pullets by food restriction during rearing. Brit. Poult. Sci., 30, 161-173.

Hocking P., Zaczek V., Jones E., MacLeod M., 2004. Different concentrations and sources of dietary fibre may improve the welfare of female broiler breeders. Brit. Poult. Sci., 45, 9-19.

Houston D.C., 1997. Nutritional constraints on egg production in birds. Proc. Nutr. Soc., 56, 1057-1065.

Kaiya H., Furuse M., Miyazato M. Kangawa K., 2009. Current knowledge of the roles of ghrelin in regulating food intake and energy balance in birds. Gen. Comp. Endocrinol., 163, 33-38.

Kostal L.C., Savory J., Hughes B.O., 1992 Diurnal and individual variation in behaviour of restricted-fed broiler breeders. Appl. Anim. Behav. Sci., 32, 361-374.

Lamosová D., Mácajová M., Zeman M. Mózes S., Jezová D., 2003. Effect of in ovo leptin administration on the development of Japanese quail. Physiol. Res., 52, 201-209.

Leterrier C., Constantin P., Le Bihan Duval E., Marche G., Nys Y., 1998. Troubles locomoteurs et qualité osseuse chez les volailles de chair. INRA Prod. Anim., 11, 125-130.

Leveille G.A, Romsos D.R, Yeh Y.Y, O'Hea E.K, 1975. Lipid biosynthesis in the chick. A consideration of site of synthesis, influence of diet and possible regulatory mechanisms. Poult. Sci., 54, 1075-1093.

MacLeod M.G., Savory C.J., McCorquodale C.C., Boyd A., 1993. Effects of long-term food restriction on energy expenditure and thermoregulation in broiler-breeder fowls (Gallus domesticus). Comp. Biochem. Physiol. Comp. Physiol., 106, 221-225.

Menge H., 1967. Effect of different sources of protein on reproduction in the hen. Poult. Sci., 46, 1528-1531.

Miura T.K., Maruyama S., Shimakura H. 2006. Neuropeptide Y mediates ghrelininduced feeding in the goldfish Carassius auratus. Neurosci. Lett., 407, 279-283.

Mondal M.S., Date Y., Yamaguchi H., Toshinai K., Tsuruta T., Kangawa K., Nakazato M., 2005. Identification of ghrelin and its receptor in neurons of the rat arcuate nucleus. Regul. Pept., 126, 55-59.
Nir I., Waites G.M., Cunningham F.J., 1975 Obesity induced by force-feeding and accompanying changes in body temperature and fertility in the male domestic fowl. Brit. Poult. Sci., 16, 505-515.

Pearson A., Herron K., 1981. Effects of energy and protein allowances during lay on the reproductive performance of broiler breeder hens. Brit. Poult. Sci., 22, 227-239.

Puterflam J., Merlet F., Faure J.M., Hocking P.M., Picard M., 2006. Effects of genotype and feed restriction on the time-budgets of broiler breeders at different ages. Applied Anim. Behav. Sci., 98, 100-113.

Reddy R.P.K., Sajadi A., 1990. Selection for growth and semen traits in the poultry industry: What can we expect in the future? In: Control of fertility in domestic birds. Brillard J.P. (Ed) INRA Editions, Paris, France, 47-59.

Renema R, Robinson F., Proudman J., Newcombe M., McKay R., 1999a. Effects of body weight and feed allocation during sexual maturation in broiler breeder hens. 1. Growth and carcass characteristics. Poult. Sci., 78, 619-628.

Renema R., Robinson F., Proudman J. Newcombe M., McKay R., 1999b. Effects of body weight and feed allocation during sexual maturation in broiler breeder hens. 2. Ovarian morphology and plasma hormone profile. Poult. Sci., 78, 629-639.

Retterstol K., Tran T.N., Haugen T.B., Christophersen B.O., 2001. Metabolism of very long chain polyunsaturated fatty acids in isolated rat germ cells. Lipids, 36, 601-606.

Richards M., Poch S., Coon C., Rosebrough R., Ashwell C., McMurtry J., 2003. Feed restriction significantly alters lipogenic gene expression in broiler breeder chickens. J. Nutr., $133,707-715$

Robertson K.M., Schuster G.U., Steffensen K.R., Hovatta O., Meaney S., Hultenby K., Johansson L.C., Svechnikov K., Söder O., Gustafsson J.A., 2005. The liver X receptor- $\beta$ is essential for maintaining cholesterol homeostasis in the testis. Endocrinology, 146, 25192530 .

Saldanha C.J., Walters B.J., Fraley G.S. 2010, Neurons that co-localize aromatase- and kisspeptin-like immunoreactivity may regulate the HPG axis of the Mallard drake (Anas platyrhynchos). Gen. Comp. Endocrinol., 166, 606-613.

Sauveur B., 1988. Reproduction des volailles et production d'œufs. Editions Quae, Paris, France, 470p.

Savory C.J., Maros K., 1993. Influence of degree of food restriction, age and time of day on behaviour of broiler breeder chickens. Behav. Proc., 29, 179-190.

Savory C.J., Hocking P.M., Mann J.S., Maxwell M.H., 1996. Is broiler breeder welfare improved by using qualitative rather than quantitative food restriction to limit growth rate? Anim. Welf., 5, 105-127.

Savory C.J., Mann J.S., 1999. Stereotyped pecking after feeding by restricted-fed fowls is influenced by meal size. Appl. Anim. Behav. Sci., 62, 209-217.

Schreihofer D.A., Golden G.A., Cameron J.L., 1993. Cholecystokinin (CCK)-induced stimulation of luteinizing hormone (LH) secretion in adult male rhesus monkeys: examination of the role of $\mathrm{CCK}$ in nutritional regulation 
of LH secretion. Endocrinology, 132, 15531560.

Sirotkin A.V., Grossmann R., María-Peon M.T., Roa J., Tena-Sempere M., Klein S., 2006. Novel expression and functional role of ghrelin in chicken ovary. Mol. Cell Endocrinol., 26, 15-25.

Shi Z.D., Shao X.B., Chen N., Yu Y.C., Bi Y.Z., Liang S.D., Williams J.B., Taouis M., 2006. Effects of immunisation against leptin on feed intake, weight gain, fat deposition and laying performance in chickens. Brit. Poult. Sci., $47,88-94$.

Song Y., Wang C., Wang C., Lu L., Chen Y., Zuo Z., 2009. Exogenous leptin promotes the recovery of regressed ovary in fasted ducks. Anim. Reprod. Sci., 110, 306-318.

Sun J., Richards M., Rosebrough R., Ashwell C., McMurtry J., Coon C., 2006. The relationship of body composition, feed intake, and metabolic hormones for broiler breeder females. Poult. Sci., 85, 1173-1184.

Tolkamp B.J., Sandilands V., Kyriazakis I., 2005. Effects of qualitative feed restriction during rearing on the performance of broiler breeders during rearing and lay. Poult. Sci., 84 , 1286-1293.

Tosca L., Crochet S., Ferré P., Foufelle F., Tesseraud S., Dupont J., 2006. AMP-activated protein kinase activation modulates progesterone secretion in granulosa cells from hen preovulatory follicles. J. Endocrinol., 190, 8597.

Voitle R., Walter J., Wilson H., Harms R., 1973. Effect of low protein and skip-a-day grower diets on subsequent performance of turkey breeder hens. Poult. Sci., 52, 543-548.

Walzem R.L., Simon C., Morishita T., Lowenstine L., Hansen R.J., 1993. Fatty liver hemorrhagic syndrome in hens overfed a purified diet. Selected enzyme activities and liver histology in relation to liver hemorrhage and reproductive performance. Poult. Sci., 72, 1479-1491.

Walzem R., Davis P., Hansen R., 1994 Overfeeding increases very low density lipoprotein diameter and causes the appearance of a unique lipoprotein particle in association with failed yolk deposition. J. Lipid Res., 35, 1354-1366.

Wehrman M.E., Welsh T.H. Jr, Williams G.L., 1991. Diet-induced hyperlipidemia in cattle modifies the intrafollicular cholesterol environment, modulates ovarian follicular dynamics, and hastens the onset of postpartum luteal activity. Biol. Reprod., 45, 514-522.

Wilson H., Harms R., 1986. Performance of broiler breeders as affected by body weight during the breeding season. Poult. Sci., 65, 1052-1057.

Wren A.M., Small C.J., Abbott C.R., Dhillo W.S., Seal L.J., Cohen M.A., Batterham R.L., Taheri S., Stanley S.A., Ghatei M.A., Bloom S.R., 2001. Ghrelin causes hyperphagia and obesity in rats. Diabetes, 50, 2540-2547.

Yu M., Robinson F., Charles R., Weingardt R., 1992. Effect of feed allowance during rearing and breeding on female broiler breeders. 2 . Ovarian morphology and production. Poult. Sci., 71, 1750-1761.

\section{Résumé}

Chez les oiseaux, comme toutes autres espèces, la nutrition et plus particulièrement le métabolisme énergétique influence la fonction de reproduction. Les modèles d'oiseaux hyperphagiques ont permis de montrer l'effet négatif dans les deux sexes d'une suralimentation, alors qu'une restriction alimentaire pendant leur croissance augmente les taux de ponte et la durée de la période fertile. Cependant les mécanismes qui sous-tendent les relations entre la nutrition, le métabolisme énergétique et la fonction de reproduction sont mal connus. Ces changements du statut nutritionnel et métabolique peuvent être transmis à l'axe de la reproduction (hypothalamus, hypophyse et gonades) aussi bien par les modifications des concentrations hormonales en insuline, adipocytokines, etc., que par celles des substrats énergétiques eux mêmes : glucose, acides gras et acides aminés. Nous rappellerons brièvement ici les effets des différents composants présents dans l'alimentation et les conséquences sur la reproduction. Les propositions d'utiliser un rationnement qualitatif seront également abordées : existe-t-il des méthodes alternatives impliquant une restriction qualitative plutôt que quantitative?

\section{Abstract}

\section{Diet, welfare and male and female bird fertility: the complex relationship}

In birds, as in other species, nutrition and energy influence the function of reproduction. The hyperphagic models have shown the negative effect of over-feeding in both sexes, and the potential rescue by food restriction treatment which can increase the length of the fertile period. However, the mechanisms underlying the relationship between nutrition, energy metabolism and reproductive functions are poorly understood. These changes in metabolic and nutritional status can be transmitted to the gonadotrope axis (hypothalamus, pituitary and gonads) by changes in hormone levels (insulin, adipokines...), or directly by the energy substrates (glucose, fatty acids and amino acids). Briefly, we discuss the effects of different components of the diet on fertility. Finally, we discuss alternative methods involving qualitative restriction rather than quantitative methods on feeding in order to improve animal welfare and in particular by reducing the behaviour of frustration and hunger.

BRIERE S., BRILLARD J.-P., PANHELEUX M., FROMENT P., 2011. Alimentation, fertilité et bien-être des oiseaux reproducteurs domestiques : des liens complexes. In : Bien-être du poulet de chair. Dossier, INRA Prod. Anim., 24, $171-180$. 
